available; $54.5 \%$ (6/11) being urgent and not available. Customers had to go to more than one pharmacy to get PMs in $20 \%$ of surveys. Descriptive data suggests pharmacies were found to be friendly and helpful.

Conclusions Despite the small sample, geographical restriction and study limitations the customer survey appeared acceptable. Our findings suggest further improvements should be made to enable access to PMs from community pharmacies even when commissioned services exist. Further research is needed to explore factors that facilitate or inhibit timely access to PMs from community pharmacies.

Acknowledgements This research was funded by Pharmacy Research UK (Grant reference GA10). The views expressed are those of the authors and not necessarily those of Pharmacy Research UK.

\section{THE HIDDEN CO-WORKER: RESEARCH REVEALING UNMET SUPPORT NEEDS IN CARERS OF PEOPLE WITH DEMENTIA WHO RECEIVE CANCER TREATMENT}

J Hopkinson, M King, R Milton. Cardiff University

\subsection{6/bmjspcare-2018-ASPabstracts.33}

Background Internationally there is a growing population of elderly people. Cancer and dementia are diseases primarily of old age. An increasing number of people will face the challenge of comorbid dementia and cancer.

Patients with dementia are diagnosed with cancer at a later stage of disease, receive less treatment and have poor survival compared to patients without dementia. The reasons are unknown. In part, because there has been no research investigating how the cancer team manage the support needs of people with dementia.

Method Our research was of case study design. Data was collected using ethnographic methods. Observation was of the clinical management of 33 people with dementia and/or memory problems in four clinics at a cancer centre. A sub-set of ten clinical consultations were recorded and seven patients were interviewed, six accompanied by a carer. This paper will report cross-case thematic analysis of findings relating to dementia carers.

Results Clinicians rely on patient/carer disclosure to identify patients with memory problems, as memory health is not documented in cancer records. Carers facilitate communication between cancer clinician and patient. They typically elect not to disclose memory problems. This has implications for safe and effective treatment. The education and support needs of carers go unrecognised, with just one example in our data of a clinician talking with the carer about how to manage the behavioural and psychological symptoms of dementia if exacerbated by a new pain medication.

Conclusion This is the first research to evidence dementia carers are hidden coworkers in the cancer team. They facilitate successful cancer management, yet their support needs are largely unrecognised. Training in dementia care is available to cancer clinicians. This should include advice on how to identify and support individuals with memory problems during treatment and recognise then respond to the needs of their carer.

\section{EXPLORING CAREGIVERS' EXPERIENCES OF CARING FOR MEN WITH ADVANCED PROSTATE CANCER IN A RESOURCE-POOR SETTING: A QUALITATIVE SERIAL INTERVIEW STUDY}

Yakubu Salifu, Kathryn Almack, Glenys Caswell. University of Nottingham, University of Hertfordshire

\subsection{6/bmjspcare-2018-ASPabstracts.34}

Background Family caregivers who assume the responsibility of care for patients living with advanced diseases could have challenges especially in resource-poor settings. The bulk and responsibility for care are predominantly provided by the family caregivers within the informal sector. The family caregivers, therefore, could be described as the 'general practitioners' of the patients at home. Using a qualitative study, the experiences of the family caregivers caring for men with advanced prostate cancer were explored.

Methods Using serial qualitative approach, 23 in-depth individual and dyad interviews were conducted. Patients and their caregivers participated in repeat interviews approximately 2 months apart, across a period of up to 6 months. Transcribed interviews were analysed using thematic analysis.

Findings This paper focuses on the experiences of family caregivers in the light of inadequate resources for care. Two main themes emerged from the data: Challenges and burden of care, and the support and coping strategies adopted. The family caregivers expressed the overburden nature of the care, 'feeling of being alone in the middle of a deep sea', disruption of their lives, and unhappiness and the fear of an unknown future. On the other hand, they rely on the support of the social network of the extended family, their faith in God, and the positive aspect of living helped to mitigate some of the challenges. The belief in reciprocity and 'giving back' through caring for their loved ones were highlighted by participants.

Conclusion Generally, the informal social network helped in the care at home. However, the magnitude of the adverse changes in their lives and challenges associated with the caring role was dependent on factors such as the level of care required, financial and other resources available, and the coping mechanisms in place. Efforts to enhance existing social support systems are recommended.

\section{Education and training | Posters 8-33}

\section{DRUG OF THE MONTH - A SNAPSHOT TRAINING TOOL AND QUICK REFERENCE GUIDE}

Kate Nolan, Ruth Hardwick. Marie Curie

\subsection{6/bmjspcare-2018-ASPabstracts.35}

Background Gaps in the knowledge of various professionals within the hospice were identified regarding medications for various reasons including, new drugs available, new uses or routes for existing drugs, unlicensed drugs as well as new members of staff unfamiliar with palliative care.

Aim To provide a training tool that would cater for all members of the multidisciplinary team and be able to be delivered 